\title{
METODOLOGÍAS PARA EL ESTUDIO DE LA COMPETENCIA MEDIÁTICA EN PROFESIONALES DE LA COMUNICACIÓN
}

\author{
Metodological tools for the Media Literacy research in \\ Mass Media professionals
}

\begin{abstract}
Autores: BUITRAgo ALONSO, Alejandro y GARCÍA MATILLA, Agustín
Becario FPI (Formación de Personal Investigador) y miembro del grupo GICAVH del Área de Comunicación Audiovisual y Publicidad de la Universidad de Valladolid. Campus Público "María Zambrano" de Segovia. Plaza Alto de los leones, 1. 40005 Segovia. alexbuitrago.alonso@gmail.com
\end{abstract}

Catedrático de Universidad, Decano de la Facultad de Ciencias Sociales, Jurídicas y de la Comunicación de la Universidad de Valladolid e Investigador Principal del Grupo GICAVH - Campus Público “María Zambrano” de Segovia. Plaza Alto de los leones, 1.40005 Segovia. agustingmatilla@gmail.com

\section{Resumen}

Vivimos tiempos en los que la Sociedad de la Información ha mutado y ya no podemos hablar de medios de comunicación estancos, sino de un contexto hipermedia que nos rodea y afecta durante las 24 horas del día. En este sentido, la evaluación de la competencia mediática en los propios profesionales de la comunicación se torna imprescindible. Por tanto, el presente artículo describe el diseño de una muestra de profesionales de la comunicación que intente albergar el mayor número posible de perfiles atendiendo específicamente a tres de sus sectores (información, ficción y publicidad) e integrando tanto los medios de comunicación tradicionales (prensa, radio, TV) como los nuevos medios, en plena ebullición y constante cambio, que conforman ese sector hipermedia.

Una vez presentado el diseño de la muestra, el siguiente paso recae en la elección de la herramienta metodológica más precisa para medir nuestro objeto de estudio, en este caso la percepción del nivel de competencia mediática presente hoy en día en el ámbito de los profesionales de la comunicación. De este modo, la siguiente parte del artículo se centra en la elaboración de un guión de "entrevista en profundidad” como metodología a seguir en este proyecto nacional de I+D puesto en marcha desde 2010 para evaluar, entre otros sectores, la competencia mediática en el sector profesional de la comunicación en nuestro país.

\begin{abstract}
We are living in a time when the information society has mutated greatly. Nowadays, we cannot speak anymore about isolated communication media but a hypermedia context, which surrounds and affects us at all times. Correspondingly, the assessment of media competition in the communication professionals has become mandatory. Hence, the present article describes the design of a sample of communication professionals. We attempt having the largest number of media profiles, specifically according to their professional expertise, namely information, fiction or marketing. To achieve our aim we need to integrate the traditional media (press, radio and TV) with a number of other novel ever-changing media, which currently constitute the hypermedia sector.
\end{abstract}

Once the sample selection is shown, the next step consists of choosing the most accurate methodological tool to gauge our subject of study: the perception of the Media Literacy level in today's communication professionals. Thus, the following part in our article is focused on developing an "in-deep interview" script. This will be the methodology to follow in our Research \& Development National Project, which has been conducted since 2010, and whose ultimate objective is the Media Literacy assessment among the communication professionals within our country.

\section{Palabras clave}

Competencia mediática, profesionales de la comunicación, entrevista en profundidad, hipermedia, alfabetización mediática

\section{Key words}

Media Competence, Mass media proffesionals, in-deep interview, hypermedia, Media Literacy 


\section{Introducción}

Desde 2011, el grupo de investigación COMPECAV, formado por miembros de diferentes Universidades que abarcan prácticamente la totalidad del territorio nacional, se encuentra trabajando en el proyecto coordinado $\mathrm{I}+\mathrm{D}+\mathrm{i}$ "La competencia en comunicación audiovisual en un entorno digital. Diagnóstico de necesidades en tres ámbitos sociales: los profesionales de la comunicación, el ámbito universitario y el ámbito de la educación obligatoria" (EDU2010-21395C03), financiado directamente por el Ministerio de Economía y Competitividad.

El grupo COMPECAV se encuentra a su vez dividido en tres equipos cuyas sedes se localizan en la Universidad Pompeu Fabra de Barcelona, Universidad de Huelva y Universidad de Valladolid (Campus "María Zambrano" de Segovia). Cada subgrupo se encarga de uno de los tres ámbitos de estudio de la "competencia en comunicación audiovisual” que conforman la investigación primaria. En el caso del equipo de Barcelona su área de estudio se centra en la evaluación de la competencia mediática en el ámbito universitario (COMPECAV-EU). El equipo de Huelva, por su parte, se encarga de los diferentes niveles que componen la enseñanza obligatoria en el sistema educativo español (COMPECAV-EO). Y, por último, el equipo coordinado desde la UVa en Segovia asume la parte de la investigación que afecta al estudio de la competencia mediática en los propios profesionales de la comunicación (COMPECAV-PC).

En este sentido, el análisis de la competencia mediática sobre los propios profesionales de los medios añade un plus de complejidad sobre la hipótesis inicial, pues no sólo se trata de llevar a cabo el estudio de una competencia que se presupone debiera estar ampliamente desarrollada en todo aquel trabajador del sector de la comunicación, sino además conocer la percepción de estos profesionales sobre la propia viabilidad de promover una educación en competencia mediática que incluya a los propios productores y a los ciudadanos en su conjunto. A su vez, el mundo de la comunicación se encuentra hoy en día notablemente diversificado y diseminado debido al contexto hipermedia en plena ebullición que nos rodea, por lo que se hacía necesario dividirlo de entrada en tres de sus más relevantes sectores respecto a la pretensión buscada en el acto comunicativo: información, ficción y publicidad.

A partir de ahí, el reto consistía en diseñar una muestra que fuera capaz de reunir del modo más completo posible la realidad actual del universo comunicativo que nos rodea y establecer un guión de "entrevista en profundidad" que nos permitiese analizar nuestro objeto de estudio, la competencia en comunicación audiovisual, de la manera más eficaz posible. Es por ello que este artículo se plantea como una reflexión sobre la metodología de investigación que sustenta este subproyecto I+D del ámbito comunicativo y los pasos efectuados hasta dar con su elección.

En la propia investigación se está desarrollando también una propuesta de metodología didáctica en asignaturas concretas del curriculum de un grado en comunicación que en este caso no es objeto de este artículo.

\section{Objetivos}

A lo largo de las páginas que componen el presente artículo se pretende mostrar el procedimiento seguido hasta dar con la elección de las herramientas metodológicas precisas para el análisis de la competencia mediática en profesionales de la comunicación.

En primer lugar, se mostrarán los pasos dados hasta la delimitación del diseño definitivo que compone la muestra de la investigación. Así pues, se llevará a cabo un recorrido sobre la premisa inicial de la que partíamos, los obstáculos encontrados en el camino y la presentación final del diseño muestral definitivo a través de una tabla de categorías cruzadas.

Una vez delimitado el diagrama definitivo sobre el que situar a los sujetos que van a componer nuestro objeto de estudio, pasaremos a exponer el guión de la principal herramienta metodológica que articula nues- 
tra investigación sobre profesionales de los medios: "la entrevista en profundidad".

El objetivo no es otro que el de presentar a la comunidad científica el work in progress de un proyecto de investigación de carácter nacional que se lleva aplicando desde 2011 sobre profesionales de medios nacionales y locales, generalistas y especializados, públi-

\section{Metodología}

Para la exposición de los objetivos anteriormente descritos se ha tomado la investigación como un camino con diversas bifurcaciones en el que a veces hay que retroceder sobre nuestros pasos para retomar la senda que verdaderamente desemboque en la consecución de nuestros propósitos.

De este modo, se narrará el período de elaboración de la muestra deteniéndonos en otras posibilidades de diseño metodológico que se descartaron y las razones por las que se decidió cambiar a la estructura metodológica actual.

Por su parte, se presentará dicho diseño muestral definitivo a través de un diagrama en el que se muestran los sectores de la comunicación sobre los que se sustenta el estudio (información, ficción y publicidad) cruzados con cada uno de los medios de comunicación analizados, ya sean medios tradicionales (prensa, ra- cos y privados; que se pretende replicar a corto plazo más allá de nuestras fronteras; y que, a día de hoy, ya se puede hablar de su eficacia contrastada a la hora de medir su objeto central de estudio: la percepción del nivel de competencia mediática que existe desde el sector profesional de la comunicación en España.

\section{Elaboración de la muestra}

En el desarrollo original de la investigación se decidió que el número de sujetos que conformarían la muestra de profesionales de la comunicación sobre los que llevar a cabo el estudio sería de 100. A partir de esta premisa, el objetivo consistía en elaborar un diseño muestral que lograra albergar el mayor número posible de perfiles pertenecientes al sector de la comunicación dentro de una justificación metodológica que huyera de la selección por azar.

En primer lugar, se optó por consultar alguna fuente fiable de datos de consumo mediático en España. Por lo tanto, se acudió al Estudio General de Medios que la AIMC (Asociación para la Investigación en Medios de Comunicación) lleva a cabo tres veces al año en las llamadas "oleadas" y que, actualmente, es el medio más fiable para datar el consumo que se hace a nivel estatal de sectores como el radiofónico o los medios impresos. De esta manera, al distribuir dio, TV) o la categoría denominada nuevos medios que incluye todo lo referente a Internet, dispositivos móviles, y en general a todo lo que rodea al sector hipermedia en constante transformación. En dicha tabla, se podrá ver también las diferentes franjas de edad delimitadas para la selección de sujetos (hasta 35 años / entre 35 y 50 / mayores de 50) y el número de profesionales a entrevistar en cada categoría hasta alcanzar los 120 que actualmente componen la muestra.

En cuanto a la exposición del guión de "entrevista en profundidad" se desglosarán uno por uno los 5 bloques que componen su estructura, describiendo qué se pretende obtener con cada uno de ellos, mencionando preguntas concretas que componen su contenido y explicando a qué faceta del estudio de la competencia mediática atañe cada apartado. los 100 sujetos de nuestra investigación en función de los datos de consumo mediático de nuestro país encontrábamos una justificación lógica para el diseño de la muestra. No obstante, nos surgía otra serie de problemas aparejados a la elección del EGM como fuente, tales como:

- El EGM no mide datos del sector de la publicidad.

- Para aparecer en el EGM hay que estar asociado al AIMC (con la consiguiente exclusión de los medios que no lo están).

- La utilización del dato de "consumo" como único baremo para medir el contexto comunicativo actual de España, cayendo de este modo en una orientación que corría el riesgo de primar sólo la presencia de los grandes medios convencionales a la hora de abordar la investigación; es decir, el 
hecho de primar únicamente a los medios "de mayor alcance": diarios de tirada nacional, canales y emisoras generalistas, etc.

- La supresión en nuestro estudio de todos aquellos medios que no alcanzan cotas de consumo suficientes como para entrar entre los 100 seleccionados. Lo que vendríamos a llamar como medios "de menor alcance": prensa y televisión local, revistas especializadas, pequeñas emisoras, etc.

- La exclusión de todos aquellos nuevos perfiles profesionales que han surgido a raíz de la revolución multimedia e hipermedia y que van más allá de los meros datos de consumo de Internet que aparecen en el informe EGM. Sería, como ya se ha comentado, lo que vendríamos a denominar como "nuevos medios" y haría referencia no sólo a la red sino también a dispositivos móviles, el fenómeno de las redes sociales, etc.

- Los datos del EGM varían de oleada en oleada (cada cuatro meses), lo que daría una vigencia extremadamente corta a la justificación sobre la que se sustentaría nuestro diseño muestral.

Una vez puesta sobre la mesa esta serie de contras llegamos a la conclusión de que empleando el EGM como única fuente para nuestro diseño muestral estaríamos apenas analizando la punta de la pirámide de la realidad mediática del país y no intentando adentrarnos en todo el espectro comunicativo de una manera global.

A partir de este convencimiento, se decidió modificar el baremo principal sobre el cual articular la muestra, esto es, huir del concepto de "representatividad real" de los profesionales del sector a través de los datos de consumo mediático y buscar el modo de incluir el mayor número de perfiles posibles independientemente de su visibilidad mediática o sus cotas de seguimiento.

Dentro de esta nueva línea, y tras contrastar con otros colegas expertos en materia de metodología cualitativa para las ciencias sociales, se decidió buscar un modelo que tuviera sentido en sí mismo, un diseño con una justificación válida desde el punto de vista metodológico que lograra representar, en mayor o menor medida, la magnitud total de esa pirámide que conforma la realidad mediática del país.
En primera instancia, se tomó conciencia de la imposibilidad de reflejar en la investigación la totalidad de perfiles profesionales existentes en la actualidad en el panorama comunicativo, dada la limitación de las 100 entrevistas en profundidad planteadas en la concepción del proyecto. Un número, 100, que es al mismo tiempo amplio y ambicioso desde el punto de vista de la herramienta metodológica elegida, la "entrevista en profundidad", y a su vez limitado a la hora de reflejar todos y cada uno de los diferentes roles que podemos encontrar hoy en día en el espectro mediático español.

Pero no se trata de detenernos en incluir uno por uno todos los diversos tipos de profesionales de la comunicación que pueden cohabitar en la Sociedad de la Información, sino de crear un modelo razonado que no excluya ningún área de relevancia de la realidad mediática. Es decir, y haciendo un símil geográfico, no se trata de incluir en la investigación un sujeto de cada localidad del país, pero sí al menos unos pocos representantes de cada provincia que conforma el conjunto a estudiar.

Finalmente, decidimos aumentar el número de sujetos de la muestra en 20 individuos $(\mathrm{N}=120)$ dada la diferente presencia de los tres sectores de la comunicación investigados (información, ficción y publicidad) en el panorama mediático. El ámbito de la "publicidad", a pesar de su radical importancia como motor social y mediático de la realidad comunicativa, no engloba en porcentaje un número de profesionales tan amplio como los sectores de información y ficción/entretenimiento, por lo que se decidió romper la estructura en tercios iguales planteada inicialmente y reducir el número de sujetos del sector publicitario incluidos en la muestra.

De esta manera, la muestra final se fijó finalmente tal que así:

- 50 profesionales del sector informativo

- 50 profesionales del sector de ficción

- 20 profesionales del sector de la publicidad

Una vez efectuada la división de sujetos para la muestra se definió gráficamente la estructura de este nuevo modelo, cuya esencia radica en el contraste de categorías a través de una tabla que cruza por un lado los tres sectores estudiados (información, fic- 
ción y publicidad) con cada uno de los cuatro subgrupos dedicados a englobar los diferentes medios de comunicación (medios impresos, radio, TV y nuevos medios). A su vez, en la tabla se reflejan también: las tres franjas de edad en que se subdivide a los profesionales del estudio (hasta 35 años / entre 35 y 50 / mayores de 50), el número entre paréntesis de sujetos a entrevistar en cada categoría y la división cometida entre medios "de mayor alcance" y "de menos alcance" dedicada a no excluir de la investigación a aquellos medios de nuevo cuño o menos visibilidad mediática.

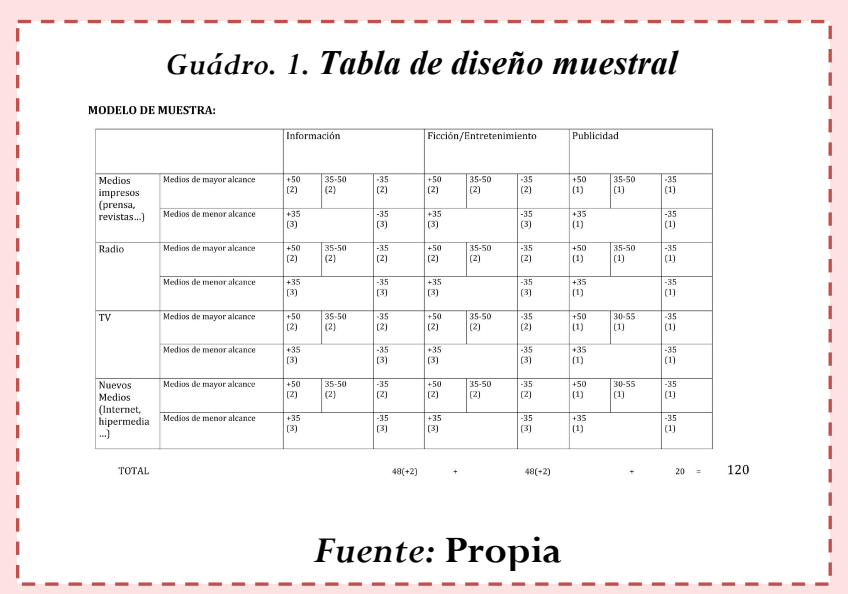

\section{Guión de entrevista en profundidad}

Una vez definido el diseño muestral definitivo el siguiente paso era estructurar la herramienta metodológica sobre la que se sustenta el proyecto: la "entrevista en profundidad".

La principal diferencia respecto al resto de investigaciones en "educación en medios" radicaba en que en este caso no se trataba de evaluar el nivel de "competencia mediática" de un colectivo en particular, sino de la percepción que tienen los propios profesionales de la comunicación sobre el nivel de alfabetización mediática existente hoy en día en la profesión.

Desde un primer momento se tuvo claro que el objeto de la investigación no podía ser "examinar" a los trabajadores de los medios sobre sus conocimientos en materia de comunicación, incluyendo su conocimiento de los lenguajes, las técnicas y las formas de explotación de los contenidos mediáticos y de los actuales sistemas de información y comunicación, pues, como es lógico, la reacción normal hubiera sido el sentirse de alguna manera "agredidas" al cuestionárseles sobre un campo sobre el que eran especialistas.

De este modo, se decidió dividir el guión de entrevista en profundidad en los 5 bloques de contenido que se presentan a continuación:

\subsection{Bloque 1. De contacto.}

En este bloque estamos interesados en la trayectoria profesional del entrevistado desde su período de formación. De manera concreta, se busca que el entrevistado hable de ese período de formación previo a la fase del ejercicio profesional y que describa su trayectoria laboral hasta la actualidad.

\subsection{Bloque 2. De uso/consumo de medios y sistemas de información y comunicación.}

En este bloque se investiga cómo se plantea el profesional su propia dieta mediática. Se pretende que el entrevistado valore la cantidad de horas aproximadas de consumo diario que dedica a los medios de comunicación y a otras tecnologías de la información como Internet, móvil, etc. Interesa saber si sería capaz de distinguir entre un uso estrictamente relacionado con la profesión y otro más específico para el ocio. También si trabaja en una jornada laboral más o menos computable en horas concretas. Y, de ser así, si el consumo de medios y sistemas de información que realiza se limita a esa jornada laboral o se extiende fuera de ella. Es además en este apartado en el que se le pregunta por el tipo de contenidos que busca en Internet.

Nos interesa que los entrevistados se manifiesten específicamente sobre la publicidad y valoren su función actual, desde la perspectiva de meros consumidores y sobre aspectos como ¿Cuándo cree que resulta más eficaz un mensaje publicitario?

\subsection{Bloque 3. Relacionado con el concepto de Alfabetización y Competencia Mediática.}

Este es uno de los bloques esenciales de la entrevista. Se intenta averiguar cómo empezó el entrevistado a usar las nuevas tecnologías y si recibió algún 
tipo de formación. Se le pregunta además por los profesionales en general, ¿cree que están suficientemente formados para el uso de medios y sistemas de información y comunicación? En cuanto a los ciudadanos, ¿cree que están suficientemente formados para leer y usar dichos medios y sistemas de información y comunicación? Y por último se incide de manera directa en el concepto a evaluar. Se le pregunta qué cree necesario enseñar para promover la competencia mediática y digital de los ciudadanos, y qué contenidos considera fundamentales para dar contenido al concepto de "competencia mediática”. Buscando siempre no sólo la respuesta en sí del entrevistado, sino principalmente su argumentación libre y espontánea.

\subsection{Bloque 4. De la percepción del nivel de Competencia Mediática en la profesión y el papel educador de los medios}

El propio título del bloque habla por sí solo de lo que se pretende en este apartado de la entrevista. Por tanto, al entrevistado se le pregunta directamente sobre qué percepción tiene del nivel de Competencia Mediática de sus compañeros, es decir, del nivel existente hoy en día de alfabetización en medios en el sector profesional de la comunicación y sobre qué papel que deben jugar los medios de comunicación en el proceso de alfabetización o educación en Competencia Mediática de la población en general.

Se pretende averiguar de esta forma, el nivel de saturación en aquellas ideas que nos servirían para poder tener un dibujo aproximado de las percepciones de los profesionales sobre sus propios colegas.

\subsection{Bloque 5. Relacionado con los ejes o di- mensiones concretas de la Competencia Me- diática}

El presente bloque hace mención directa a los ejes de la Competencia Mediática delimitados por Ferrés (2007) con la colaboración de un equipo de exper- tos en «La competencia en comunicación audiovisual: propuesta articulada de dimensiones e indicadores». Pueden verse los resultados de esta investigación en Ferrés, J; García Matilla, A; Aguaded, J.I; Fernández Cavia, J. Figueras, M. y Blanes, M. (2011). En ambas publicaciones se definían, de manera concreta, las seis dimensiones: tecnología, lenguaje, producción y programación, recepción e interacción, ideología y valores $\mathrm{y}$, por último, la estética. Dicho documento ha sido recientemente revisado por Ferrés y Piscitelli (2012) reafirmando de este modo el valor de las seis dimensiones y sus respectivos indicadores.

Por tanto, a lo largo de este bloque se lleva a cabo un recorrido por cada uno de los ejes incidiendo en qué importancia le dan a dicho componente de la Competencia Mediática, qué formación poseen en dicha dimensión y en qué grado la han aplicado en su posterior dedicación profesional. A su vez, se le pregunta por qué tipo de conocimientos considera esenciales dentro de ese ámbito a la hora de formar al ciudadano como consumidor crítico.

\subsection{Bloque 6. Análisis DAFO.}

En este bloque se sitúa al entrevistado en una hipotética propuesta de un programa para la educación en Competencia Mediática en la actualidad. Como el propio análisis DAFO indica, se trata de averiguar por un lado qué "debilidades y amenazas" y por otro qué "fortalezas y oportunidades" ve el entrevistado para el desarrollo de la educación en Competencia Mediática en el momento actual.

Por último, y tras la consecución de los 6 bloques, se le entrega una ficha al entrevistado en la que debe rellenar tanto sus datos personales como el medio en el que trabaja actualmente, así como un breve recorrido por su trayectoria profesional (medios en los que ha trabajado y cargo).

\section{Conclusiones}

Resulta innegable que los profesionales de la comunicación juegan un papel clave no sólo como creadores y difusores de los mensajes de los medios, sino también como formadores de la ciudadanía y bién como formadores de la ciudadanía y responsables de la creación de una sociedad madura, crítica y competente a la hora de interpretar los impactos mediáti- 
cos que recibe cada ciudadano durante las 24 horas del día.

Llegados a este punto, es igualmente incuestionable que a la hora de llevar a cabo un análisis sobre el nivel de Competencia Mediática presente en la sociedad actual, uno de los pilares sobre los que se asienta nuestra investigación es en el estudio de las personas que trabajan precisamente en su sector de la comunicación. No se pueden descifrar, analizar y entender los mensajes lanzados por los medios sin entender a quienes los idean, producen, editan y transmiten. Del mismo modo, no podemos pretender educar a la ciudadanía en comunicación mediática si no somos capaces de transmitir a los profesionales su importancia trascendental como educadores, en primera instancia, de los ciudadanos a los que van dirigidos sus mensajes.

Es, por tanto, una necesidad absoluta contar con profesionales formados en Ciencias de la Información/Comunicación, expertos en todo lo referente a alfabetización mediática y competencia digital, y comprometidos con su labor de crear una ciudadanía crítica y competente en medios. Sin ese requisito es imposible que se creen las condiciones necesarias para promover una comunicación útil a la ciudadanía y comprometida con el mandato tácito que la sociedad otorga a los profesionales para reforzar la democracia en un contexto de libertad y progreso mutuo.

La investigación aquí presentada forma parte del macroproyecto $\mathrm{I}+\mathrm{D}+\mathrm{i}$ "La competencia en comunicación audiovisual en un entorno digital. Diagnóstico de necesidades en tres ámbitos sociales: los profesionales de la comunicación, el ámbito universitario y el ámbito de la educación obligatoria" (EDU2010-21395C03), que analiza el actual contexto de la formación en comunicación, los currícula de estudios en los niveles básicos de enseñanza y en los niveles universitarios y también en el ámbito profesional.

Nuestra investigación, en su actual fase de evolución como work in progress, está descubriendo cómo la opinión de los profesionales se puede clasificar de una manera casi dicotómica entre aquellos que ven como una necesidad prioritaria una educación en materia de comunicación o en competencia comunicativa de la propia ciudadanía que incluye la exigencia de actualización de los profesionales y otra parte que se muestra sumamente insensible y escéptica ante cualquier propuesta que plantee esa posible formación generalizada de la población.

Con este artículo se ha querido exponer el work in progress de esa ambiciosa investigación que se está llevando a cabo con profesionales de la comunicación de diversa índole y que tratan de reproducir la realidad comunicativa de nuestro país en sus diferentes sectores. Estamos esperanzados en que la producción científica resultante de dicho estudio y de todo el macroproyecto marcará un importante avance en el devenir de la investigación en Competencia Mediática (Media Literacy) y supondrá el punto de partida de nuevas propuestas y líneas de investigación con las que continuar trabajando en este apasionante reto que es la Educación en Medios.

\section{Apoyos}

Estudio enmarcado en la Convocatoria de Proyectos I+D del Ministerio de Economía y Competitividad con clave: EDU2010-21395-C03-02, titulado "Los profesionales de la comunicación ante la competencia en comunicación audiovisual en un entorno digital”.

\section{Referencias}

- Aguaded, J.I., García Matilla, A. y Ferrés, J. (2012). La competencia mediática de la ciudadanía española: competencias y retos. Icono 14, Vol. X, no 3, pp. 23-42.
- Ferrés, J; García Matilla, A; Aguaded, J.I; Fernández Cavia, J. Figueras, M. y Blanes, M. (2011). Competencia Mediática. Investigación sobre el grado de competencia de la ciudadanía en España. ITEMEC. 
- García Matilla, A., Navarro, E. y Orozco, G. (2012). Educational challenges in times of mass self-communication: a dialogue among audiences. En Yearbook 2013 of the International Clearinghouse on Children, Youth and Media. Publica Nordicom (The Nordic Information Centre for Media and Communication Research) and UNITWIN Network, UNESCO.
- Ferrés, J. (2007) La competencia en comunicación audiovisual: Dimensiones e indicadores, Comunicar, vol. XV, N²9, pp. 100-107.

- Ferrés, J. y Piscitelli, A. (2012) La competencia mediática: propuesta articulada de dimensiones e indicadores. Comunicar, vol.XIX, No38, pp. 75-82

\section{Forma de Citación}

BUITRAGO ALONSO, Alejandro y GARCÍA MATILlA, Agustín: Metodologías para el estudio de la competencia mediática en profesionales de la comunicación. Revista Communication Papers, $\mathrm{N}^{\circ} 2$, Monográfico 1, páginas 75 a 82. Departamento de Filología y Comunicación de la Universidad de Girona. Recuperado el _ de___ de 2 ___ de: http://www.communicationpapers.es 